\title{
Libros digitales para la educación universitaria en
}

\section{América Latina}

\author{
Juan José Prieto Gutiérrez \\ Doctor; Universidad Complutense de Madrid, Madrid, Espanha; \\ jujpriet@ucm.es
}

\begin{abstract}
Resumen: La presencia del libro digital en la educación universitaria es una realidad. El objetivo principal del estudio es confirmar el desarrollo del libro digital en las 50 universidades de America Latina que mejor están situadas según el ranking de Scimago durante el año 2016. También se busca determinar el tipo de acceso al documento y las plataformas más empleadas. Como tercer objetivo se examinan las ventajas y desventajas que el personal de las bibliotecas experimenta día a día con el paulatino incremento de los libros digitales. Con el fin de responder a los objetivos planteados se consultan las páginas Web de las 50 instituciones analizadas y se envían encuestas a los responsables de los centros. Los diversos análisis confirman que todas las bibliotecas estudiadas ofrecen libros digitales a los usuarios universitarios. El $50 \%$ de las instituciones prefiere comprar el derecho de acceso a las obras mientras que el $40 \%$ combina la suscripción y la compra, siendo las plataformas más empleadas Springer y Wiley eBooks.
\end{abstract}

Palabras clave: Libros digitales. Tecnologías de la educación. Educación superior. Fuentes de información.

\section{Introducción}

El nacimiento de las primeras universidades desarrolló inevitablemente la creación de bibliotecas que, en primeras instancias, solo consultaban los profesores de las instituciones con el objeto de aprender y el deseo de transmitir sabiduría y conocimiento. Los documentos librarios depositados en las bibliotecas han sido desde hace varios siglos los instrumentos de trabajo más empleados en las instituciones educativas universitarias, ofreciendo soporte a las actividades de docencia e investigación. 
Las diversas evoluciones de las bibliotecas a lo largo del siglo XX no han detenido el uso de los materiales en formato papel, habiendo incluso condicionado el desarrollo de variadas instituciones bibliotecarias. Pero, desde finales del siglo XX, con el surgimiento de Internet, el progreso tecnológico y de las redes informáticas han generado nuevos desafíos para las bibliotecas; fortaleciéndolas y favoreciendo el servicio que prestan a la comunidad estudiantil.

Las universidades, por exigencia de la sociedad, están implementando modelos educativos flexibles soportados por el empleo de tecnologías de la información y la comunicación (TIC) en el aprendizaje. Estos modelos se basan en incrementos de programas de enseñanza online y semipresenciales, en donde el autoaprendizaje y el e-learning cobran cada vez más protagonismo así como la generación de redes virtuales de todos los colectivos de la universidad. Un ejemplo clarificador se aprecia en los recursos digitales y los nuevos soportes de lectura y consulta de las bibliografías dispuestas en los centros educativos (LEMKEN, 1999). Los usuarios de las bibliotecas, estudiantes universitarios, docentes e investigadores son partícipes de la transformación de los soportes bibliotecarios. Estos, para acceder a la información han ido sustituyendo el aula y la biblioteca, como espacios físicos por contextos virtuales (ALONSO; CORDON; GOMEZ, 2011). Siendo capaces, ahora, de enviar, almacenar, editar y leer documentos en un soporte diferente al papel. (LAM et al., 2009; ASHCROFT, 2011).

Desde finales de los años 90, del siglo XX, debido a la paulatina popularización de Internet los textos online se han convertido en una herramienta habitual de consulta y aprendizaje tanto para profesores e investigadores como para estudiantes de las universidades de todo el mundo (TUROFF; HILTZ, 1982). Cabe destacar que las publicaciones periódicas fueron los documentos que comenzaron las transformaciones del formato papel al electrónico. Desde entonces, las ediciones online se han afianzado como pilares de la docencia e investigación (KERKA, 1996).

Una vez asentadas las revistas electrónicas en las instituciones educativas superiores, las monografías y manuales científicos han sido una de las últimas 
incorporaciones documentales como soporte de enseñanza y aprendizaje, favorecidos por el desarrollo de las tecnologías de la comunicación, los sistemas de edición digital y el cambio en los hábitos de lectura y adquisición (CULL, 2011), destacando la posibilidad de compra a perpetuidad, suscripción a paquetes o pago por uso.

Es paradójica la desigual evolución que han mantenido las revistas y las monografías en su transformación desde soportes "tradicionales" a soportes electrónicos. Esta, ha obedecido al propio destino de las diversas publicaciones.

Aquellas investigaciones que presentan un ciclo de vida corto premian los formatos online. Así, las publicaciones periódicas electrónicas ofrecen resultados divulgativos de una forma rápida y ágil, ya que, dependiendo de las materias, la inmediatez en la divulgación y su dinámico análisis es necesario para poder situarse en la posición adecuada.

En cambio, los libros y manuales suelen ofrecer un contenido que, muchas veces, no requieren de una pronta lectura, no existiendo un temor a la interrupción o la desactualización de la obra. Incluso la propia extensión del documento conlleva una consulta más pausada y sistemática en el tiempo.

\section{El libro digital en América Latina}

La presencia del libro digital en universidades sigue creciendo en todo el mundo. Por ejemplo, según el Ebook Usage in U.S. Academic Libraries referente al año 2016 la media de ebooks en los centros de Estados Unidos de América (EE.UU.) ha aumentado los últimos años ininterrumpidamente (EBOOK..., 2016). Del año 2012 al 2016 ha incrementado un $40 \%$ de 35.500 a 47.183 ebooks. En EE.UU. en el año 2012 las bibliotecas universitarias invertían un $30 \%$ en libros digitales. Se estima que para 2017 la inversión aumente al 60\%. En España, se pasará del 2\% al 15\% o 20\% (GIL; CELAYA, 2015). En otro mercado influyente, como son las universidades de Gran Bretaña, la encuesta más reciente realizada por la Sociedad Británica de Bibliotecas Universitarias y Nacionales (SCONUL) indica que durante el año 2004-05 cada 
usuario solo tenía un solo libro digital, a su disposición, para su consulta. En cambio, durante el curso 2003-04 disponían de noventa por usuario (2015).

América Latina no se queda atrás y ante el reto de mejorar la calidad de la oferta educativa universitaria se ha embarcado en el gran desafío de ofrecer, desde las bibliotecas generalmente, innumerables manuales básicos y documentos científicos en formato digital, destinados a estudiantes y profesores.

Indudablemente, desde la aparición las (TIC) en la región, allá por los años 80, se asentaron cómodamente con el objetivo de favorecer la adquisición de competencias y de habilidades cognitivas de aquellos que acuden a los centros educativos (SUNKEL; TRUCCO; ESPEJO, 2014).

Ya en la propuesta del Manifiesto sobre el Libro Electrónico, redactado por la UNESCO y por el Centro Regional para el Fomento del Libro en América Latina y el Caribe, CERLALC, se sugería la transición desde el libro físico hacia el libro electrónico, con el objetivo de garantizar una inserción de los países del entorno a las actuales y nuevas tendencias tecnológicas (2011).

En materia de educación, el Plan de Acción sobre la Sociedad de la Información para América Latina y el Caribe (eLAC2015), redactado por CEPAL en el año 2010, sugería el empleo de las TIC como instrumentos de desarrollo e inclusión social, presentando una prioridad: universalizar el acceso y expandir el uso de las TIC para la educación (CEPAL, 2010).

La existencia de políticas TIC ha sido, y es, una condición necesaria para la integración de tecnologías digitales en las instituciones educativas de América Latina. La práctica totalidad de los países del continente mantiene políticas formales en este área, que se iniciaron a inicios de en los años 2000 con la instalación de infraestructuras tecnológicas en los centros educativos (SUNKEL, 2006).

Con el paso de los años, esta situación ha originado que las universidades de la región posean espacios, laboratorios o salas con computadoras y equipos informáticos para uso de alumnos y docentes, desde donde es posible consultar la amplia oferta de materiales bibliográficos en formato electrónico. Como es sabido, la propia esencia del documento digital permite ser consultado fuera de las fronteras institucionales, por lo que se 
ofrecen accesos remotos a los contenidos previa identificación del usuario universitario.

Con unas infraestructuras en constante cambio, los recursos digitales presentes en las instituciones de educación superior de América Latina comenzaron a extenderse y, durante el año 2014, incrementaron en un 1,5\% respecto al año anterior. Cabe destacar que las editoriales universitarias crecieron un 9,6\% (CERLALC, 2015).

En definitiva, esta investigación pretende ofrecer la situación, a fecha de 2016, del libro en formato digital en las principales universidades de América Latina. La muestra en estudio comprende 50 universidades de Latinoamérica situadas en los primeros puestos del ranking Scimago.

Por tanto, este trabajo plantea un triple objetivo; por un lado, el objetivo principal del estudio es confirmar la presencia del libro digital en las más importantes bibliotecas universitarias de América Latina. Como segundo objetivo se busca determinar el tipo de acceso al documento y cuales son las plataformas más empleadas, en las universidades examinadas, para acceder a los libros digitales. Por último y como tercer objetivo se examinan las ventajas y desventajas que el personal de las bibliotecas experimenta día a día con el paulatino incremento de los libros digitales.

\section{Medología}

Se describe la metodología aplicada para el conocimiento de los libros digitales en una selección de universidades de Latinoamérica. Para obtener una muestra relevante de las instituciones se adoptó el criterio de elegir los 50 primeros centros educativos situados durante el año 2016 en el ranking de Instituciones de Scimago (ver Apéndice A). Los datos fueron extraídos durante el mes de septiembre del citado año.

El SCImago Institutions Rankings es una clasificación de instituciones académicas clasificadas por un indicador compuesto que combina tres conjuntos diferentes de indicadores, basados en el rendimiento de la investigación, los productos de innovación y el impacto social medido por su visibilidad en la 
Web. Toda la información obtenida se basa en datos de Scopus de Elsevier (BORNMANN; DE MOYA-ANEGÓN; LEYDESDORFF, 2011).

La recopilación de datos, a fin de responder a los objetivos planteados, y conocer el proceso transformación que están padeciendo las universidades analizadas a la hora de ofrecer documentación e información a sus usuarios para el desarrollo óptimo de sus aprendizajes y trabajos, se ha realizado a través de una consulta de las páginas Web de las bibliotecas pertenecientes a las instituciones objeto de estudio, por ser estas quienes ponen a disposición el material académico (en Apéndice A se presentan las páginas Web revisadas).

Es importante destacar que la consulta rigurosa de gran parte de la colección digital requiere identificación previa, limitándose exclusivamente a los usuarios miembros de la institución. Debido a ello y desde una posición externa, por parte del autor, a la totalidad de los centros investigados es imposible conocer el conjunto total de títulos que oferta cada biblioteca, al igual que diferentes clasificaciones ordenadas por materias, autor, título, etc. Este conocimiento solo es posible una vez permitida la entrada a las plataformas o agregadores, con previa identificación personal del usuario.

Ante esta situación, con la pretensión de responder a los objetivos fijados y ampliar el conocimiento de la colección de libros digitales dispuestos por las universidades analizadas, se ha recopilado más información mediante la aplicación de encuestas a las 50 universidades (ver Apéndice B). La encuesta está formada por preguntas abiertas, siendo enviadas por correo electrónico, a partir del segundo semestre del año 2016, a las bibliotecas objeto de estudio. La tasa de respuesta fue del $78 \%$, al contestar 39 personas.

Con el deseo de arrojar una mayor claridad sobre los objetivos planteados se establecen diferentes preguntas en la encuesta, las cuales buscan determinar si los libros digitales están presentes en las universidades analizadas; las ventajas y desventajas que presentan respecto a los libros en formato papel; las plataformas que emplean para la consulta de los documentos; el modelo de compra y selección de los materiales; el porcentaje del presupuesto destinado a la compra de obras digitales y su variación año tras año. 


\section{Resultados}

En primer lugar, se constata que las 50 universidades analizadas ofrecen a sus usuarios (profesores e investigadores y estudiantes) acceso a libros electrónicos. Para corroborar esta posición, ya intuida a priori, se han examinado la totalidad de las páginas Web de las bibliotecas universitarias objeto de estudio. En este primer examen, realizado directamente sobre los portales de las 50 instituciones, se ha comprobado que todas las universidades ofrecen acceso online a recursos educativos. Es importante distinguir las dos modalidades de consulta que ofrecen a los usuarios. Por un lado, mediante un acceso acotado a los beneficiaros del centro, sobre aquel acervo generalmente de pago y por tanto excluido a personas externas a la institución y por otro lado, un acceso abierto a ciertos repositorios institucionales y gratuitos y a la producción científica propia.

En el conjunto de centros analizados se presenta idéntica forma de acceso a la información disponible en las respectivas bases de datos, bien desde computadoras dispuestas en la red institucional o bien desde el exterior, previa identificación con el nombre de usuario y contraseña para validar el acceso remoto.

Por tanto, la posibilidad de consulta de monografías académicas y científicas, por parte de los lectores de cada universidad, se realiza desde la Web de cada institución bien enlazando con las plataformas de préstamo de libros electrónicos, agregadores, sobre los documentos que forman los repositorios institucionales o bien a través del, cada vez más común, acceso abierto a textos académicos (BADINI, 2011).

El análisis del contenido digital de las 50 universidades ofrece más información. Todas disponen de sus propios servicios de publicaciones en donde enriquecer e impulsar el repositorio institucional de la propia universidad, formado por artículos, investigaciones, libros, imágenes, multimedia, etc. Otra semejanza radica en que una gran parte del material bibliográfico que componen las colecciones de los centros educativos proviene de licencias de acceso de editores comerciales. 
La gran cantidad de repositorios digitales dispuestos en las universidades analizadas, que promueven el autoarchivo, demuestran el avance significativo del acceso abierto. Las dos principales listas de repositorios a nivel mundial son el Directorio de Repositorios de Acceso Abierto (OpenDoar) y el Registro de Repositorios de Acceso Abierto (ROAR), en donde se ofrecen, respectivamente, la existencia de 311 y 399 repositorios en América Latina. Todos ellos gestionados por instituciones educativas y científicas.

En los inicios del movimiento internacional del acceso abierto ya se demostraban las mejoras de la capacidad científica en los países y se favorecía la disminución de la brecha informativa para docentes, investigadores y estudiantes universitarios (CHAN; KIRSOP; ARUNACHALAM, 2005).

Con el fin de disponer de una mayor información y profundizar en la situación del mercado digital en las universidades de Latinoamérica, se envió, una encuesta a la totalidad de los centros analizados como se ha comentado. Gracias a los resultados recibidos es posible responder los objetivos segundo y tercero que se plantean en este trabajo.

En cuanto al segundo objetivo del trabajo que es la determinación de las plataformas digitales de agregación de contenidos y de comercialización que se sitúan en las universidades analizadas.

A continuación se ofrece una figura en donde visualizar la presencia de las plataformas online por el número de universidades que las utiliza.

Figura 1 - Plataformas digitales más frecuentes

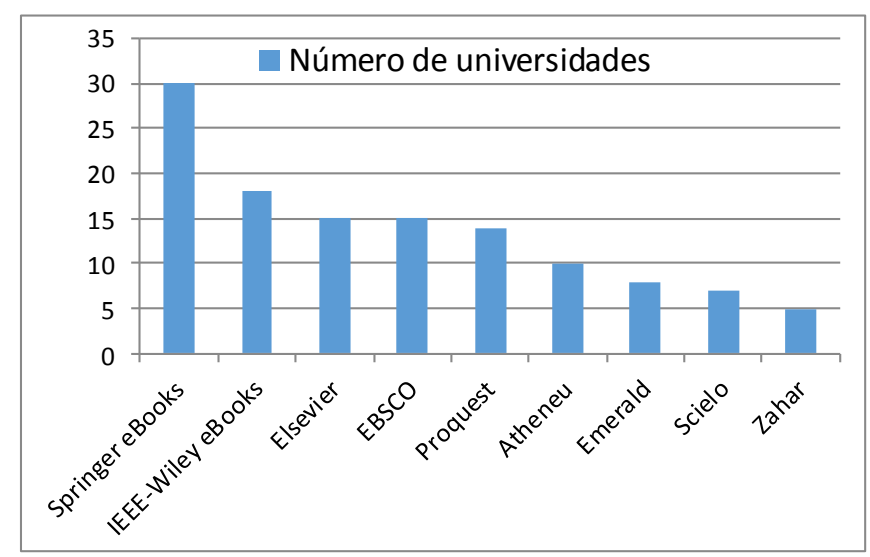

Fuente: elaborado por el autor a partir de la encuesta destinada a las bibliotecas. 
Con el fin de responder al objetivo segundo se ofrece la figura anterior. Una vez realizado el análisis de cada biblioteca se observa la presencia de diferentes editoriales comerciales, plataformas, agregadores y repositorios institucionales responsables de ofrecer a los usuarios de cada universidad los libros digitales. Los editores más importantes del mundo están presentes en Latino América, como dato destacado, 30 de las universidades estudiadas disponen de colecciones digitales de Springer eBooks. En segundo lugar se sitúan las colecciones de IEEE-Wiley eBooks, presentes en 18 centros; muy de cerca se sitúan las plataformas de Elsevier y EBSCO (en 15 universidades) y Ebrary de ProQuest en 13 centros.

Esta coyuntura hace imposible identificar, con exactitud, el número total de libros digitales que las universidades ponen a disposición de sus usuarios. El contraste entre repositorios institucionales, agregadotes o bases de datos de pago conlleva a una problemática para poder calcular el número de documentos disponibles en cada momento. A veces, el mercado online genera que repositorios, de acceso público en Internet, sean ofrecidos como "propios" a los usuarios.

En la misma línea del acceso a los materiales educativos online, la encuesta nos reporta el modelo de negocio de compra de libros digitales en las universidades analizadas. Del total de centros analizados se dispone que 20 instituciones prefieren la compra a perpetuidad (representa el 51\%), 4 los modelos de suscripción (10\%) y 15 combinan ambas modalidades (39\%).

Figura 2 - Modelo de negocio de compra de libros

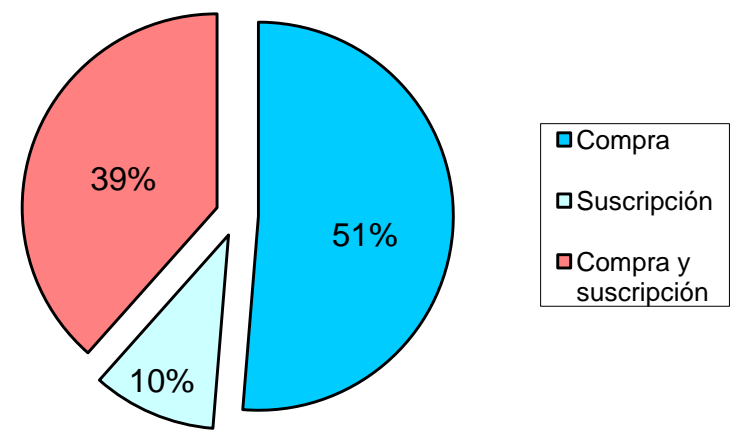

Fuente: elaborado por el autor a partir de la encuesta destinada a las bibliotecas. 
La compra a perpetuidad es la opción más cara, pero garantiza el acceso al documento y se ahorra las renovaciones periódicas. El modelo de suscripción a colecciones o paquetes es ventajoso cuando se busca formar una colección de una forma rápida, pero cuando la institución ya dispone de obras es una opción no muy elegida porque la colección incluye algunas obras de poca o nula demanda. Ninguno de los centros analizados ha indicado el modelo de pago por uso.

El resultado del tercer objetivo se obtiene a través de la encuesta realizada, en donde se presentan las ventajas y desventajas que los profesionales de la comunidad universitaria Latinoamericana perciben de los libros digitales respecto al sistema tradicional de consulta de monografías en soporte físico, reflejando las sensaciones actuales. A continuación se ofrecen las respuestas obtenidas más relevantes.

Ventajas que ofrecen los libros electrónicos: Acceso permanente 24/7; versiones multiusuarios, permitiendo acceso simultáneo de varios estudiantes; posibilidad búsqueda en el texto, edición, envío de información, incorporación de elementos multimedia, integración de imagen y video, gestión de protección de derecho y propiedad intelectual, etc.; los dispositivos de lectura, con amplia capacidad de almacenamiento, responden a la demanda, en el que la ergonomía, la portabilidad y la movilidad son elementales para el usuario; la proliferación de ebooks se adapta a los actuales planes de estudio y a las exigencias de los estudiantes; ahorro en reposición de ejemplares y encuadernación; descongestión y liberación de espacio en estanterías y zonas de trabajo; liberación de espacio físico.

Desventajas: diversidad de formatos y los sistemas de seguridad Digital Rights Management (DRM); dificultad de acceso para estudiantes sin computador. Dependencia de Internet. Ancho de banda limitado, debiendo reducir el volumen o peso de ciertos documentos; necesidad de formación ante niveles de conocimiento escaso. Poco hábito de lectura; pocos libros en español. Reducida colección de títulos en ese idioma; alto costo de adquisición de libros de forma individual. Algunas editoriales ofrecen paquetes o colecciones grandes y costosas; modalidad de suscripción no permite acceso perpetuo; la modalidad 
de compra requiere, en ocasiones, adquirir varias licencias. Resultando, a veces, más costosas que las versiones impresas; en algunas ocasiones, los equipos jurídicos de la universidad no aceptan licencias de las bibliografías electrónicas.

La información recibida en algunas respuestas reflejan cierta obviedad, como la posibilidad de consultar los materiales las veinticuatro horas del día o el ahorro de espacio frente a los formatos físicos, pero hay ciertas desventajas que es posible solventar mediante cursos de formación o video tutoriales, en el caso de los niveles de conocimiento escaso. Inquietudes globales se presentan en los formatos y modalidades de los modelos de negocio. Las bibliotecas deben analizar detenidamente los modos de licencias: si compran a perpetuidad, si prefieren el modelo de suscripción a paquetes o incluso si prefieren el pago por uso. En esta tendencia, editores y consorcios podrían colaborar para constituir un mercado electrónico para ofrecer licencias de libros digitales directamente a las bibliotecas. Este deseable contexto ofrecería políticas beneficiosas por parte de los editores hacia las bibliotecas universitarias y científicas.

Otra de las preguntas realizadas en la encuesta, colateral a los objetivos de este trabajo, confirma la situación actual, comentada anteriormente, la totalidad de los bibliotecarios y personas que respondieron la encuesta fueron tajantes al señalar que año tras año se incrementan los títulos en las bibliotecas, así como conocimiento y uso.

La inversión digital de las universidades analizadas se desprende del resultado de la encuesta realizada. En la actualidad, la proporción de la inversión dedicada a la compra de obras digitales es baja comparada con el soporte papel, pero los datos suministrados por las bibliotecas son muy interesantes. 


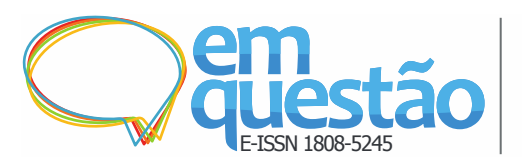

Libros digitales para la educación universitaria en

América Latina

Juan José Prieto Gutiérrez

Figura 3 - Porcentaje del presupuesto, relativo a adquisiciones, dedicado a obras digitales

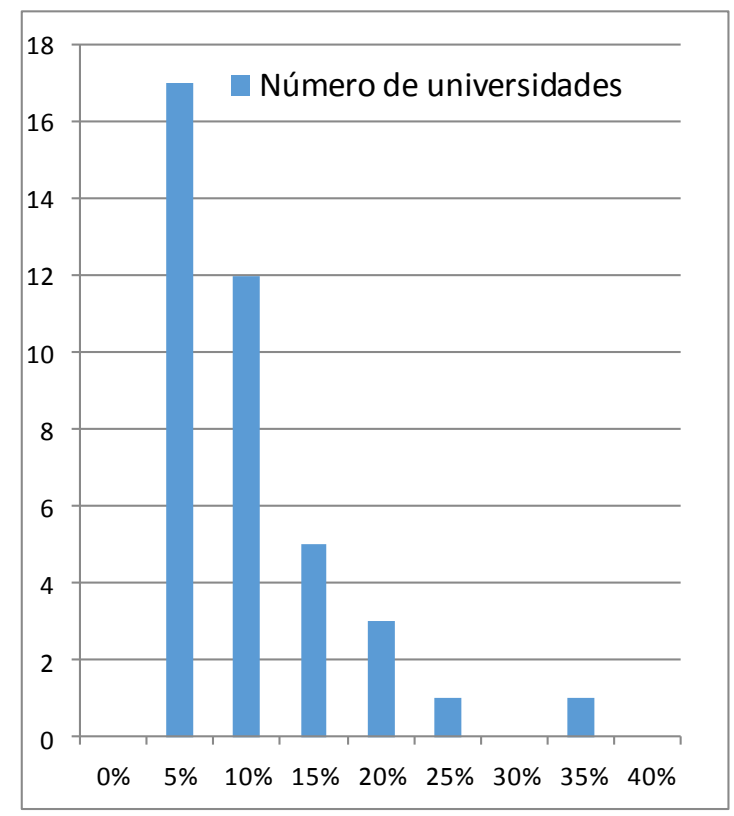

Fuente: elaborado por el autor a partir de la encuesta destinada a las bibliotecas.

Aproximadamente, el $80 \%$ de los centros que respondieron dedican entre el $5 \%$ y el $15 \%$ del presupuesto al formato digital (de una forma ilustrativa se presenta en la figura 3). En la misma línea, 36 de los 39 centros que respondieron a la encuesta afirmaron que la tendencia en los próximos años es hacia el aumento de la inversión en la compra de libros digitales. En cambio, los 2 restantes indicaron que la tendencia se situaba en torno a un mantenimiento del gasto. Estos datos suponen que el $95 \%$ de los centros aumentará las compras dirigidas a la colección digital.

\section{Discussión y conclusiones}

La situación descrita demuestra la paulatina integración del libro electrónico en las bibliotecas universitarias de América Latina, lo que implica una declaración de intenciones a los actuales contextos de aprendizaje, donde lo online adquiere una vital importancia, aprovechado por las grandes extensiones geográficas que presenta el continente americano. 
Diversos estudios en el ámbito universitario demuestran que la existencia de libros digitales disponibles en la institución demanda interés por parte de los usuarios, si bien, las pautas de consulta difieren al soporte papel. El uso en línea suele emplearse para navegar y realizar una comprobación rápida generalmente (SHIN, 2011) y para lecturas de pocas hojas. Tales comportamientos todavía están influenciados por rutinas adquiridas con las revistas científicas (GARCÍA; ALONSO; MARTIN, 2010).

La cultura digital ha impactado en las universidades, indicando una aceptación en el contexto de la educación superior e indudablemente la integración de los mismos en la vida académica.

El uso de Internet crece a ritmos amplios y su relevancia implica la presencia en la vida diaria. En la misma línea, se aprecia cómo el crecimiento de los dispositivos móviles (laptops, tabletas y teléfonos) en la actual sociedad ha favorecido la proliferación de formatos electrónicos en las universidades. Ya que contribuyen a la adaptación de las cada vez más frecuentes clases virtuales en detrimento de las sesiones magistrales.

De cualquier modo, esta situación se preveía hace varios años, generando en la actualidad un paulatino asentamiento del mercado digital. No cabe duda que tanto la calidad como la cantidad de los libros electrónicos seguirán creciendo y su impacto en la circulación de las bibliotecas se asentará, como está ocurriendo en otras zonas geográficas.

Con todo, la progresiva integración de libros electrónicos en bibliotecas universitarias de Latinoamérica, no se está realizando de la misma forma. La disparidad de plataformas documentales y contenidos implica diferencias presupuestarias e inversiones en este ámbito, e incluso desconfianzas relacionadas con la integración de los contenidos en las universidades.

En el corto plazo y con el fin de superar algunas de las desventajas planteadas por algunas bibliotecas, en relación al libro electrónico, sería necesario realizar planes de formación en todas las escalas, tanto a estudiantes como a profesores, con el fin de obtener esa capacitación necesaria para el manejo de los soportes virtuales. Por otro lado, el análisis de las páginas Web de las 50 bibliotecas demuestra la necesidad global de activar políticas de difusión 
adecuadas y planes de marketing con el deseo de llegar a la mayor parte de los usuarios. En muchos casos es ardua e intensa la búsqueda de los contenidos científicos y académicos por las plataformas, ya que la visibilidad de la producción y actividad es reducida. En este sentido, una adecuada publicidad y estructura de las páginas Web facilitaría la consulta de la documentación.

\section{Agradecimientos}

Agradecer a los revisores por su aportación al artículo. Sus recomendaciones han mejorado los aspectos formales y de contenido sustancialmente.

\section{Referências}

ALONSO-ARÉVALO, Julio; CORDÓN-GARCÍA, José Antonio; GÓMEZ-DÍAZ, Raquel. El libro electrónico en la biblioteca universitaria y de investigación. Biblios: Revista de Bibliotecología y Ciencias de la Información, [S.1.], n. 42, 2011. Disponible en: <https://biblios.pitt.edu/ojs/index.php/biblios/article/view/7/37>. Acceso el: 15 ene. 2017.

ASHCROFT, Linda. Ebooks in libraries: an overview of the current situation. Library Management, [S.1.], v. 32, n. 6/7, p. 398-407, 2011.

BABINI, Dominique. Acceso abierto a la producción científica de América latina y el Caribe: identificación de principales instituciones para estrategias de integración regional. Revista Iberoamericana de Ciencia, Tecnología y Sociedad CTS, Buenos Aires, v. 6, n. 17, 2011.

BORNMANN, Lutz; DE MOYA-ANEGÓN, Félix; LEYDESDORFF, Loet. The new excellence indicator in the World Report of the SCImago Institutions Rankings 2011. Disponible en: <https://arxiv.org/ftp/arxiv/papers/1110/1110.2305.pdf>. Acceso el: 15 ene. 2017. arXiv preprint arXiv:1110.2305, 2011.

CENTRO REGIONAL PARA EL FOMENTO DEL LIBRO EN AMÉRICA LATINA Y EL CARIBE, CERLALC. El libro en cifras Boletín estadístico del libro en Iberoamérica, Santafé de Bogotá, v. 7, 2015. Disponible en: <http://cerlalc.org/ellibro-en-cifras-boletin-estadistico-del-libro-en-iberoamerica/>. Acceso el: 15 ene. 2017.

CHAN, Leslie; KIRSOP, Barbara; ARUNACHALAM, Subbiah. Open access archiving: the fast track to building research capacity in developing countries. SciDev.Net, London, Nov. 2005. Disponible en: <http://hdl.handle.net/1807/4415>. Acceso el: 15 ene. 2017. 
CULL, Barry. W. Reading revolutions: Online digital text and implications for reading in academe. First Monday, Chicago, v. 16, n. 6, 2011. Disponible en:

<http://pear.accc.uic.edu/ojs/index.php/fm/article/view/3340/2985> Acceso el: 15 ene. 2017.

EBOOK Usage in U.S. Academic Libraries 2016. Library Journal, New York, 2016. Disponible en: <http://lj.libraryjournal.com/downloads/2016academicebooksurvey/>. Acceso el: 15 ene. 2017.

GARCÍA, José Antonio; ALONSO-ARÉVALO, Julio; MARTIN-RODERO, Helena. Los libros electrónicos: la tercera ola de la revolución digital. Anales de documentación, Murcia, v. 13, p. 53-80, 2010. Disponible en: <http://revistas.um.es/analesdoc/article/download/106991/101681>. Acceso el: 15 ene. 2017.

GIL, Manuel; CELAYA, Javier. Evolución y tendencias digitales en Latinoamérica. 2015. Disponible en: <http://bit.ly/1sEpW74>. Acceso el: 15 ene. 2017.

KERKA, Sandra. Distance learning, the internet, and the world wide web. ERIC Digest, 1996.

LAM, Paul et al. Usability and usefulness of eBooks on PPCs: How students' opinions vary over time. Australasian Journal of Educational Technology, Brisbane, v. 25, n. 1, p. 30-44, 2009. Disponible en:

<http://citeseerx.ist.psu.edu/viewdoc/download?doi=10.1.1.363.3830\&rep=rep 1\&type= pdf > Acceso el: 15 ene. 2017.

LEMKEN, Birgit. Ebook: the missing link between paper and screen. In: CONFERENCE ON HUMAN FACTORS IN COMPUTING SYSTEMS, 1999, Pittsburgh. Proceedings... Pittsburgh: ACM CHI 99, 1999. Disponible en: $<$ http://citeseerx.ist.psu.edu/viewdoc/download?doi=10.1.1.46.5481\&rep=rep1\&type $=p$ df>. Acceso el: 15 ene. 2017. CHI 99 Workshop on Designing Electronic Books.

CEPAL. PLAN DE ACCIÓN ELAC 2015. Plan de acción sobre la sociedad de la información y del conocimiento de América Latina y el Caribe. [S.1.]: CEPAL Naciones Unidas, 2010. Disponible en: <http://www.cepal.org/cgi-

bin/getprod.asp?xml=/elac2015/noticias/paginas/9/44209/P44209.xml\&xsl=/elac2015/t pl/p18f.xsl\&base=/elac2015/tpl/top-bottom.xsl> Acceso el: 15 ene. 2017.

REGISTRY OF OPEN ACCESS REPOSITORIES. Roar. 2017. Disponible en: <http://roar.eprints.org/>. Acceso el: 15 ene. 2017.

SHIN, Dong-Hee. Understanding e-book users: Uses and gratification expectancy model. New Media \& Society, [S.1.], v. 13, n. 2, p. 260-278, 2011.

SOCIETY OF COLLEGE, NATIONAL AND UNIVERSITY LIBRARIES, SCONUL. Changing trends in loans, visits $\&$ the use of e-books. London, 2015. Disponible en: <http://www.sconul.ac.uk/tags/sconul-statistics> Acceso el: 15 ene. 2017.

SUNKEL, Guillermo. Las tecnologías de la información y la comunicación (TIC) en la educación en América Latina: una exploración de indicadores, n. 125. [S.1.]: United Nations Publications, 2006. Disponible en: 
<http://repositorio.cepal.org/bitstream/handle/11362/6133/S0600907.pdf? sequence=1>. Acceso el: 15 ene. 2017.

SUNKEL, Guillermo; TRUCCO, Daniela; ESPEJO, Andrés. La integración de las tecnologías digitales en las escuelas de América Latina y el Caribe: una mirada multidimensional. Santiago de Chile. CEPAL, 2014. Disponible en:

<http://200.9.3.98/bitstream/handle/11362/36739/S20131120.pdf? sequence=1>. Acceso el: 15 ene. 2017.

THE DIRECTORY OF OPEN ACCESS REPOSITORIES. OpenDOAR, 2017. Disponible en: <http://www.opendoar.org/>. Acceso el: 15 ene. 2017.

TUROFF, Murray; HILTZ, Starr Roxanne, The electronic journal: A progress report.

Journal of the American Society for Information Science, Massachusetts, v. 33, n. 4, p. 195-202, 1982. Disponible en:

<https://web.njit.edu/ turoff/Papers/ElectronicJournal.html> Acceso el: 15 ene. 2017.

UNESCO Y CENTRO REGIONAL PARA EL FOMENTO DEL LIBRO EN AMÉRICA LATINA Y EL CARIBE, CERLALC. Manifiesto sobre el libro electrónico. 2011. Disponible en: 〈http://www.cerlalc.org/Manifiesto_Esp.pdf > Acceso el: 15 ene. 2017.

\section{Apéndice A - Lista de las 50 primeras universidades iberoamericanas en el}

\section{Ranking Scimago}

\begin{tabular}{|c|c|c|}
\hline & Universidad & URL \\
\hline 1 & Universidade de São Paulo & http://www5.usp.br/pesquisa/bibliotecas \\
\hline 2 & Universidade Estadual de Campinas & http://www.sbu.unicamp.br/ \\
\hline 3 & $\begin{array}{l}\text { Universidad Nacional Autónoma de } \\
\text { México }\end{array}$ & http://www.dgb.unam.mx/ \\
\hline 4 & Universidade Federal do Rio de Janeiro & http://www.sibi.ufrj.br/ \\
\hline 5 & $\begin{array}{l}\text { Universidade Estadual Paulista Júlio de } \\
\text { Mesquita Filho }\end{array}$ & http://www.unesp.br/portal\#!/cgb \\
\hline 6 & $\begin{array}{l}\text { Universidade Federal do Rio Grande do } \\
\text { Sul }\end{array}$ & https://www.ufrgs.br/bibliotecas/ \\
\hline 7 & Universidade Federal de Minas Gerais & https://www.bu.ufmg.br/bu/ \\
\hline 8 & Universidad de Buenos Aires & http://www.sisbi.uba.ar/ \\
\hline 9 & Universidad de Chile & $\begin{array}{l}\text { http://www.portaluchile.uchile.cl/biblioteca } \\
\underline{\underline{s}}\end{array}$ \\
\hline 10 & Pontificia Universidad Católica de Chile & http://bibliotecas.uc.cl/ \\
\hline 11 & Universidade Federal de São Paulo & http://bv-unifesp.bvs.br/php/index.php \\
\hline 12 & Universidade Federal de Santa Catarina & http://portal.bu.ufsc.br/ \\
\hline 13 & Universidad de Puerto Rico Río Piedras & http://biblioteca.rrp.upr.edu/ \\
\hline 14 & $\begin{array}{l}\text { Centro de Investigación y de Estudios } \\
\text { Avanzados del IPN }\end{array}$ & http://biblioteca.cinvestav.mx/ \\
\hline 15 & Instituto Tecnológico de Tijuana & http://tectijuana.edu.mx/biblioteca/ \\
\hline 16 & Universidade Federal do Paraná & $\begin{array}{l}\text { http://www.ufpr.br/portalufpr/servicos/bibli } \\
\text { otecas/ }\end{array}$ \\
\hline 17 & Universidade Federal de Pernambuco & $\begin{array}{l}\text { http://www.biblioteca.ufpe.br/pergamum/bi } \\
\text { blioteca/index.php }\end{array}$ \\
\hline 18 & Universidade Federal de São Carlos & http://www.bco.ufscar.br/ \\
\hline
\end{tabular}




\begin{tabular}{|c|c|c|}
\hline 19 & Universidad del Rosario & http://www.urosario.edu.co/biblioteca/ \\
\hline 20 & Universidade de Brasília & http://www.bce.unb.br/ \\
\hline 21 & Universidad Nacional de Colombia & http://bibliotecas.unal.edu.co/ \\
\hline 22 & Universidad Nacional de la Plata & http://biblio.unlp.edu.ar/ \\
\hline 23 & $\begin{array}{l}\text { Universidad Técnica Federico Santa } \\
\text { María }\end{array}$ & http://www.bibliotecas.usm.cl/web/ \\
\hline 24 & Universidade Federal da Bahia & $\begin{array}{l}\text { http://www.pergamum.bib.ufba.br/pergamu } \\
\text { m/biblioteca/index.php }\end{array}$ \\
\hline 25 & Instituto Politécnico Nacional & $\begin{array}{l}\text { http://www.ipn.mx/biblioteca/Paginas/inici } \\
\text { o.aspx }\end{array}$ \\
\hline 26 & Universidade Federal de Santa Maria & http://w3.ufsm.br/biblioteca/ \\
\hline 27 & Universidad Nacional de Rosario & http://bibliotecas.unr.edu.ar/ \\
\hline 28 & Universidad Nacional de Mar de Plata & http://biblio1.mdp.edu.ar/ \\
\hline 29 & Universidade Federal do Ceará & http://www.biblioteca.ufc.br/ \\
\hline 30 & Universidade Federal de Viçosa & http://www.bbt.ufv.br/ \\
\hline 31 & Universidad de los Andes Colombia & $\begin{array}{l}\text { https://biblioteca.uniandes.edu.co/index.php } \\
\text { ?lang=es }\end{array}$ \\
\hline 32 & Universidade Federal Fluminense & http://www.bibliotecas.uff.br/ \\
\hline 33 & Universidade do Estado do Rio de Janeiro & http://www.rsirius.uerj.br/novo/ \\
\hline 34 & Universidad de la República Uruguay & http://www.biur.edu.uy/F \\
\hline 35 & Universidade Federal de Lavras & http://www.biblioteca.ufla.br/ \\
\hline 36 & $\begin{array}{l}\text { Pontificia Universidade Católica de } \\
\text { Minas Gerais }\end{array}$ & $\begin{array}{l}\text { http://www2.pucminas.br/biblioteca/index } \\
\text { padrao.php }\end{array}$ \\
\hline 37 & $\begin{array}{l}\text { Pontificia Universidade Católica do Rio } \\
\text { Grande do Sul }\end{array}$ & http://biblioteca.pucrs.br/ \\
\hline 38 & Universidad Nacional de Córdoba & http://www.bmayor.unc.edu.ar/ \\
\hline 39 & Universidade Estadual de Maringá & http://www.bce.uem.br/ \\
\hline 40 & Universidade Federal de Goiás & https://www.bc.ufg.br/ \\
\hline 41 & Universidad Nacional de Rio Cuarto & http://juanfilloy.bib.unrc.edu.ar/ \\
\hline 42 & Universidad de Antioquia & $\begin{array}{l}\text { http://www.udea.edu.co/wps/portal/udea/we } \\
\text { b/inicio/sistema-bibliotecas }\end{array}$ \\
\hline 43 & $\begin{array}{l}\text { Universidade Estadual do Norte } \\
\text { Fluminense Darcy Ribeiro }\end{array}$ & $\begin{array}{l}\text { http://www.uenf.br/portal/index.php/br/bibl } \\
\text { iotecas/biblioteca-cch.html }\end{array}$ \\
\hline 44 & Universidad Autonoma de Yucatan & http://www.bibliotecas.uady.mx/ \\
\hline 45 & Universidade Estadual de Londrina & http://www.uel.br/bc/portal// \\
\hline 46 & Universidade Federal do ABC & http://portal.biblioteca.ufabc.edu.br/ \\
\hline 47 & Universidade Federal da Paraíba & http://www.biblioteca.ufpb.br/ \\
\hline 48 & $\begin{array}{l}\text { Universidade Federal do Rio Grande do } \\
\text { Norte }\end{array}$ & http://sisbi.ufrn.br/bczm/ \\
\hline 49 & Universidad de Carabobo & http://www.bc.uc.edu.ve/ \\
\hline 50 & Universidad de Concepción & http://www.bibliotecas.udec.cl/ \\
\hline
\end{tabular}

\section{Apéndice B - Encuesta a bibliotecas}

1. ¿Cuáles son las principales ventajas de los libros electrónicos científicos, sobre libros impresos? ¿Y las desventajas?

2. ¿Piensa que el libro digital o electrónico está asentado en las bibliotecas universitarias? 
3. En su biblioteca, ¿La inversión en la compra de libros digitales va en aumento año tras año?

4. ¿Qué porcentaje del presupuesto de compra de materiales se destina a obras digitales?

5. ¿Qué plataformas emplean para el préstamo de libros digitales? Springer, Elsevier, Emerald, Taylor \& Francis, Cambridge, etc.

6. ¿Cómo se accede a los libros digitales en su universidad? ¿Mediante suscripción o compra?

7. En relación a los modelos de compra y selección ¿Que suelen emplear o por qué modelo se decantan? ¿por la compra de título a título o por la compra de colecciones?

\title{
Ebooks for higher education in Latin America
}

\begin{abstract}
The presence of the university ebooks is a reality. The main objective of the study is to confirm the development of the ebook in the top 50 universities Scimago ranking in Latin America during the year 2016. It also seeks to determine the type of access to the document and the most used platforms. The third objective examines the daily advantages and disadvantages for library staff experience with the gradual increase in ebooks. In order to respond to the objectives set, the websites of the top 50 institutions analyzed were consulted and surveys were sent to the centers. The analyzes confirm that all the libraries studied offer ebooks to university users. $50 \%$ of institutions prefer to buy the right of access to the works while $40 \%$ combines subscription and buy the access. The most used platforms are Springer and Wiley eBooks.
\end{abstract}

Keywords: Ebooks. Education technology. Higher education. Information resources.

\section{E-books para a educação universitária na América Latina}

Resumo: A presença da instrução livro universitário digital é uma realidade. $\mathrm{O}$ principal objetivo do estudo é confirmar o desenvolvimento do livro digital em 50 universidades da América Latina que estão em melhor posição como classificado pela Scimago durante 2016. Ele também serve para determinar o tipo de acesso ao documento e as plataformas mais utilizadas. Como uma terceira visam as vantagens e desvantagens que a experiência pessoal da 
biblioteca todos os dias com o aumento gradual de livros digitais são examinados. A fim de cumprir os objetivos nos sites das 50 instituições analisadas são consultados e os inquéritos são enviados para os responsáveis pelos centros. As várias análises confirmam que todas as bibliotecas oferecem livros digitais estudados para os utilizadores universitários. 50\% das instituições preferem comprar o direito de acesso às obras, enquanto $40 \%$ assinatura combinados e compra, os mais utilizados Springer e Wiley plataformas de ebooks.

Palavras-chave: Livros digitais. Tecnologias educacionais. Educação superior. Fontes de informação.

Recebido em: 18/10/2016

Aceito em: 23/01/2017 